\section{VALIDACIÓN DE UNA ESCALA PARA EL ANÁLISIS DE LAS PERCEPCIONES DE LOS RESIDENTES SOBRE EL IMPACTO SOCIAL DE UN CENTRO DEPORTIVO}

\author{
VALIDAÇÃO DE UMA ESCALA PARA A ANÁLISE DAS PERCEPÇÕES DOS \\ MORADORES SOBRE O IMPACTO SOCIAL DE UM CENTRO ESPORTIVO
}

\section{VALIDATION OF A SCALE TO ANALYSE RESIDENTS' PERCEPTIONS OF THE SOCIAL IMPACT OF A SPORTS CENTRE}

\author{
Fernando Garcia-Pascual ${ }^{\star}$, David Parra-Camacho**, \\ Romulo Jacobo González-García*
}

\begin{abstract}
Palabras clave: Cambio social. Centros de acondicionamiento. Estudios de validación. Percepción social.

Resumen: La finalidad de este estudio es validar una escala para el análisis de las percepciones de los residentes sobre el impacto social de un centro deportivo. Se recogió una muestra de 406 residentes del municipio de Moncada (Valencia), con un error de muestreo de $\pm 4,82$, que contestaron a una encuesta de 31 ítems que recogían posibles impactos sociales derivados de la presencia del centro deportivo en la localidad. La aplicación de análisis factorial exploratorio y confirmatorio redujo la escala a 28 indicadores distribuidos en siete dimensiones de impacto: impacto sociocultural, impacto socioeconómico, impacto en la imagen y la promoción del municipio, impacto en el desarrollo urbano y las infraestructuras, impacto en la cohesión social, igualdad y equidad, impacto sobre la salud e impacto sobre los hábitos y niveles de actividad física. Los resultados permitieron comprobar la validez y fiabilidad de la escala propuesta para el objeto de estudio.
\end{abstract}

Palavras chave: Mudança social. Academias de ginástica. Estudos de validação. Percepção social.

Keywords: Social change. Fitness centres. Validation studies. Social perception.

Resumo: 0 objetivo deste estudo foi validar uma escala para a análise das percepções dos moradores sobre o impacto social de um centro esportivo. Se recolheu uma amostra de 406 moradores do município de Moncada (Valência) com erro de amostragem de $\pm 4,82$. Os moradores responderam a um questionário de 31 itens que incluíram possíveis impactos sociais derivados da presença do complexo esportivo na localidade. A aplicação da análise fatorial exploratória e confirmatória reduziu a escala para 28 indicadores distribuídos em sete dimensões de impacto: impacto sociocultural, impacto socioeconômico, impacto na imagem e promoção do município, impacto no desenvolvimento e infraestruturas urbanas, impacto na coesão social, igualdade e equidade, impacto na saúde e impacto nos hábitos e níveis de atividade física. Os resultados permitiram verificar a validade e confiabilidade da escala proposta para o objeto de estudo.

Abstract: The purpose of this study is to validate a scale to analyse residents' perceptions of the social impact of a sports centre. A sample of 406 residents of Moncada, Valencia, was collected with sampling error of \pm 4.82 . They responded to a 31 -item survey on potential social impacts of the presence of the sports centre in that town. Exploratory and confirmatory factorial analysis reduced the scale to 28 indicators distributed in seven impact dimensions: sociocultural; socioeconomic; image and promotion of the town; urban development and infrastructures; social cohesion; equality and equity; health; and physical activity habits and levels. The results made it possible to check the validity and reliability of the scale proposed for the object of study.
*Universidad de Valencia. Valencia, Espanha.

E-mail: fernando.garcia-pascual@uv.es; romulojgg@ hotmail.com

**Universidad Católica de Valencia

Valencia, Espanha.

E-mail: david.parra@ucv.es

Recebido em: 02-10-2018

Aprovado em: 03-06-2019

Publicado em: 26-06-2019

DOI:

https://doi.org/10.22456/1982-8918.86497

(c) (i) () Licence 


\section{INTRODUCCIÓN}

Actualmente existe cierta tendencia a medir la preocupación de la sociedad acerca de todo lo que acontece, como pueden ser el uso de instrumentos que reflejen entre otros las inquietudes, opiniones, cambios, reacciones, actitudes y percepciones para analizar las conductas de los actores sociales.

En primer lugar, el término percepción según Boone y Kurtz (2012, p. 150) es "[...] el significado que una persona atribuye a los estímulos entrantes recogidos a través de los cinco sentidos (vista, oído, tacto, gusto y olfato)". Es descrito como un proceso mediante el cual una persona selecciona, organiza e interpreta la información para crear una imagen significativa de nuestro entorno (WILMSHURST; MACKAY, 2002). De este modo, las personas tienen diferentes percepciones porque experimentan los mismos estímulos o información de diferentes maneras, en función de sus propias características y de la personalidad, y por una selección e interpretación de los diversos estímulos que varía debido a las habilidades sensoriales (REISINGER, 2009).

En cuanto al impacto, es importante destacar que se puede entender como la "huella" que dejan los constantes cambios sociales dentro de la sociedad durante un tiempo. El impacto social es definido desde el área del turismo por Hall (1992) como "[...] la manera en la que el turismo y sus efectos cambian los sistemas de valoración, el comportamiento laboral, las estructuras comunitarias y los estilos y la calidad de vida tanto a nivel colectivo como individual". Olsen y Merwin (1977), nombran el impacto social como "cambios en la estructura y funcionamiento del orden social que se produce en conjunto como una innovación ambiental, tecnológica o social".

Desde hace unos años, en el contexto deportivo, ya le otorgan importancia al impacto social al igual que el impacto económico, y es que los estamentos públicos dan la misma prioridad o más, a los costes intangibles (impacto social) que los costes tangibles (económico).

En esta diferencia entre costes tangibles e intangibles, hay que destacar el estudio de Gibson (2006) el cual señalaba que aun teniendo un alto valor los estudios relacionados con el impacto económico, dentro de la comunidad, los trabajos de impacto social tienen cada vez un valor más predominante.

Los autores Gursoy y Kendall (2006), desarrollaron y validaron un modelo estructural que midiera los impactos que perciben los residentes del estado de Utah (Estados Unidos), acerca de los Juegos Olímpicos de Invierno en el año 2002. Más actual, fue el estudio llevado por Collins et al. (2007), cuyos resultados mostraron, que el turismo deportivo que deriva en estancias a largo plazo, tiene incidencias negativas.

Como se ha comentado anteriormente, existe cierto vacío en cuanto al impacto social de instalaciones deportivas, ya que en este ámbito de aplicación, no existen estudios en cuanto a la literatura se refiere. En los últimos años, en España, se ha incrementado en un gran porcentaje la construcción de instalaciones deportivas, y como consecuencia de ello, el aumento de las actividades deportivas.

Dada la ausencia de un instrumento para medir la percepción sobre el impacto social de una instalación deportiva, para determinar las variables que podrían recoger la percepción sobre el impacto social de la instalación deportiva, se ha partido de la clasificación sobre los impactos 
propuesta por otros autores que han recopilado tanto a nivel teórico (PREUSS; SOLBERG, 2006) como empírico (AÑó; CALABUIG; PARRA, 2012; BALDUCK; MAES; BUELENS, 2011; CALABUIG et al., 2014; CHEN; TIAN, 2015; FREDLINE; JAGO; DEERY, 2003; GÓNZALEZGARCÍA et al., 2016; GURSOY et al., 2011; KAPLANIDOU; KARADAKIS, 2010; KARADAKIS; KAPLANIDOU, 2012; KIM; GURSOY; LEE, 2006; LIU, 2016; LLOPIS; GIL, 2011; NTLOKO; SWART, 2008; PARRA et al., 2016; PRAYAG et al., 2013) los factores para medir los impactos sociales de los eventos deportivos.

Si bien la naturaleza de los eventos deportivos es temporal y las instalaciones deportivas son de carácter permanente, es importante destacar que muchos de los impactos generados tanto por los eventos deportivos como por las instalaciones deportivas pueden provocar cambios perdurables en la calidad de vida de los residentes. De acuerdo con los trabajos mencionados, y teniendo presente las diferencias entre un evento deportivo y una instalación deportiva, se han destacado las siguientes dimensiones para analizar la percepción sobre el impacto social de una instalación deportiva: impacto socioeconómico, impacto sociocultural, impacto en el desarrollo urbano y en las infraestructuras, impacto en la imagen y la promoción del municipio, impacto en la cohesión social, igualdad y equidad, impacto sobre la salud e impacto sobre los hábitos y niveles de actividad física.

Las ciudades que albergan estas instalaciones deportivas obtienen beneficios sociales, principalmente a largo plazo: mejora de la salud, comprensión de la cultura, cohesión social, etc.

A nivel socioeconómico numerosos estudios han comprobado que los residentes perciben que los eventos deportivos pueden contribuir a mejorar indicadores como el empleo, el incremento de la actividad comercial, el consumo o las inversiones en la zona (LORDE, GREENIDGE; DEVONISH, 2011; PRAYAG et al., 2013). Dichos impactos pueden ser generados también por una instalación deportiva debido a las posibilidades de dinamización de la economía local ofreciendo puestos de trabajo en la instalación. También, puede contribuir a incrementar el número de negocios y comercios en torno a la instalación para dar respuesta a las demandas de consumo de los usuarios de la misma.

Desde el punto de vista sociocultural, diversos trabajos han destacado que los ciudadanos percibían que la acogida de eventos deportivos proporcionaban una oportunidad para conocer a nuevas personas, favorecer el intercambio cultural y la comprensión de otras culturas, fortalecer y conservar las tradiciones culturales locales e incrementar la solidaridad y hospitalidad de los ciudadanos con respecto a los visitantes (CHENG; JARVIS, 2010; KIM et al., 2006; MALFAS; THEODORAKI; HOULI et al., 2004; WAITT, 2003; ZHOU; AP, 2009). En muchas ocasiones las instalaciones deportivas se construyen para promover la socialización y el disfrute por un estilo de vida saludable entre los residentes de una comunidad (WAH; PYUN, 2015). Asimismo, los centros deportivos pueden contribuir a ser un punto de reunión y de acogida de nuevos residentes.

En cuanto a la cohesión social, uno de los aspectos más importante de las instalaciones deportivas en cuanto al impacto social donde está ubicada dicha instalación, es el sentimiento de pertenencia de los mismos y que sientan una identificación con la instalación (DECCIO; BALOGLOU, 2002).

Swart y Bob (2012) en su trabajo relacionado con las percepciones de los residentes de Ciudad del Cabo sobre el estadio del Mundial de fútbol celebrado en 2010, señalaron 
las razones principales por las cuales los residentes valoraban positivamente la instalación deportiva, en este caso el campo de fútbol, situado en la zona de residencia de los mismos. Los principales argumentos que indicaban los residentes consultados en este trabajo se centraban en la promoción del fútbol en la zona, contribución al desarrollo del deporte, creación de oportunidades de trabajo y el incremento de la economía local. Es importante subrayar que la mayoría de los encuestados apoyó la organización de la Copa Mundial de la FIFA 2010 y compartió un sentimiento de orgullo por este logro nacional.

Existen ciertas similitudes entre el impacto social de los eventos deportivos y el de las instalaciones deportivas, pero quizá una de las similitudes más sustanciales y a su vez de suma importancia es el legado de las instalaciones y espacios deportivos. Este factor importante dentro de la gestión del deporte, se da en gran parte, en aquellos eventos de grandes magnitudes, una vez acabado dicho evento, las instalaciones perduran y de los estamentos depende darle continuidad en su utilización. Malfas, Theodoraki y Houlihan (2004) en su trabajo explican que los grandes eventos deportivos suelen dejar legados de instalaciones que no tienen una relación directa con el evento como pueden ser centros comerciales, instalaciones de ocio cuya única finalidad es tener una mejor apariencia física de la localidad que ha albergado dicho evento. También, Brown y Massey (2004) en su trabajo acerca de los Juegos de la Commonwealth de 2002, defendían que la construcción de las infraestructuras deportivas tenía mayor beneficio para los atletas de élite que para los propios residentes de la localidad de acogida. Hiller (2006) argumenta que como consecuencia de los Juegos Olímpicos, las instalaciones deportivas como bien pueden ser velódromos, estadios, etc; pueden ser utilizadas para futuras competiciones o incluso como centros de formación, produciéndose adaptaciones de las mismas y actualizándose para cumplir con estándares mundiales.

Por otro lado, Smith y Fox (2007), destacan que ante la organización de un gran evento deportivo, surge un problema, siendo por un lado el legado que genera la construcción o mejora de las infraestructuras, y por otro lograr que los beneficios y recursos empleados se malgasten en infraestructuras innecesarias.

Desde el punto de vista del impacto sobre la salud e impacto sobre los hábitos y niveles de actividad física, algunos trabajos como el de Weed, Coren y Fiore (2009), indicaron que puede existir una relación entre el potencial de los legados de las instalaciones y el impacto en la participación deportiva o en actividades físicas de niños y jóvenes, pudiendo obtener diferentes resultados en función del acontecimiento. Wicker, Hallmann y Breuer (2013) señalaron que varios investigadores destacaban la disponibilidad de infraestructuras e instalaciones deportivas como un factor importante para la participación individual en el deporte (ATKINSON et al., 2005; DISHMAN; SALLIS; OHRENSTEIN, 1985; LIM et al., 2011). En la misma línea, Wicker, Breuer y Pawlowski (2009) destacan que para las personas que practican deporte la infraestructura deportiva adecuada es crucial para mantener la participación deportiva. Por lo tanto, parece evidente que la construcción de una instalación deportiva en una zona que carezca de este tipo de servicios puede contribuir a incrementar los niveles de práctica deportiva y de mejora de la condición física, actuando como catalizador para mejorar la salud y, por lo tanto, para la mejora de la calidad de vida de los residentes del municipio.

En este trabajo se propone un instrumento para el análisis de las percepciones de los residentes en torno al impacto social de un centro deportivo en su localidad. Así pues, el objetivo 
de este trabajo es analizar la validez y fiabilidad de una escala de carácter multidimensional para analizar las percepciones de los residentes sobre el impacto social de un centro deportivo.

\section{MÉTODO}

La población objeto de estudio de este trabajo son los residentes de la localidad de Moncada, municipio situado en la provincia de Valencia y a tan solo $7 \mathrm{~km}$ del área metropolitana. Dicha población dispone de distintos espacios deportivos tanto de carácter público como privado. Dentro de los espacios deportivos de índole privada, se encuentra el Centro Deportivo Moncada (CDM), cuya instalación es una de las más importantes de la población, por su carácter multidisciplinar. Dicha instalación fue inaugurada el 19 de Abril de 2011. Se construyó con la finalidad de ofrecer a la población un servicio nuevo y al alcance de todos los perfiles sociodemográficos. Tanto la construcción, como la gestión de la instalación es privada, que parte de una concesión administrativa por parte del ayuntamiento de la población, por ello se entiende como gestión indirecta. El centro deportivo ofrece diferentes tipologías de tarifas para poder alcanzar todos los perfiles de la sociedad (tercera edad, población activa, jóvenes, población parada, familias, etc), cada una de ellas obedece a un precio de tarifa estipulado, que el usuario mensualmente renueva.

El CDM, ofrece un servicio integral de deporte, ya que se puede encontrar desde una sala de musculación amplia y con una gran variedad de máquinas con la última tecnología, dos piscinas climatizadas para realizar actividades como aquagym o cursos de natación, 3 salas de actividades dirigidas y un SPA. EI CDM, actualmente, tiene inscritos 2.116 usuarios repartidos entre las distintas modalidades de tarifas, en función de los distintos perfiles sociodemográficos (edad, situación laboral, etc.).

\subsection{PARTICIPANTES}

La muestra de la investigación es de 407 residentes del municipio con un error de muestreo de $\pm 4,82$ para el conjunto de la población. La edad media es de 38,5 años $( \pm 15,79)$., de los cuales 211 son varones (52\%) y 195 son mujeres $(48 \%)$, encuestados en diferentes puntos de la localidad.

\subsection{INSTRUMENTO}

El instrumento partió de 31 ítems tomados de diferentes investigaciones sobre impacto social de los eventos deportivos y del turismo (AÑÓ; CALABUIG; PARRA, 2012; FREDLINE; JAGO; DEERY, 2003; KIM et al., 2006; NTLOKO; SWART, 2008). La redacción de los indicadores se adaptó a las peculiaridades de un centro deportivo.

Para asegurar la validez de contenido se consultó la opinión de tres jueces expertos en la investigación sobre el impacto social del deporte y tres gestores deportivos. Estos especialistas se encargaron de revisar el contenido, la claridad, la confiabilidad y el formato de los ítems planteados con la finalidad de evitar posibles confusiones en su interpretación y ofrecer sugerencias para la mejora de los mismos. Tras el análisis de la coherencia y el grado de adecuación de los ítems al objeto de estudio, se realizaron algunos cambios en la redacción 
de los ítems para mejorar su comprensión y dicha versión pasó a administrarse a los residentes del municipio.

\subsection{PROCEDIMIENTO}

El instrumento de medida se pasó en distintos enclaves de la población, zonas concurridas, donde se encontraron residentes de todos los perfiles sociodemográficos. Estos datos se recogieron mediante una encuesta estructurada y autoadministrada con presencia del investigador. Se utilizó un muestreo de conveniencia debido a la ausencia de un marco de muestreo adecuado y de un censo de residentes permanentes en la población, diferente de empadronados. No obstante, los entrevistadores fueron entrenados para administrar las encuestas mediante un seminario de formación y se les indico que recogieran encuestas de distintos grupos de población para lograr el mayor equilibrio posible en diversas variables sociodemográficas relacionadas con el sexo o la edad. El período utilizado para recoger los cuestionarios fue el primer trimestre de 2015.

\subsection{ANÁLISIS DE DATOS}

Los resultados obtenidos fueron sometidos a diferentes análisis estadísticos mediante el programa SPSS versión 21, el programa FACTOR (LORENZO-SEVA; FERRANDO, 2006) y el programa EQS 6.2. En primer lugar, se realizó un análisis descriptivo de los ítems de la escala, indicando las medias, desviaciones típicas, la asimetría y curtosis de cada indicador. Después, para comprobar la validez interna se realizó un análisis factorial exploratorio (AFE) y un análisis factorial confirmatorio (AFC).

En el caso del AFE se siguieron las recomendaciones de Lloret-Segura et al. (2014), utilizando el método de método de extracción de máxima verosimilitud (MV) y posteriormente una rotación Oblimin directo. Para determinar el número de factores se utilizó el procedimiento del Análisis Paralelo, mientras que para comprobar el ajuste del modelo se analizaron los coeficientes de la raíz media cuadrática residual (RMCR) y el índice gamma o GFI (TANAKA; HUBA, 1989), que deben ser inferiores al punto de corte .05 (HARMAN, 1980) y .95 (RUIZ; PARDO; SAN MARTÍN, 2010), respectivamente. Por último, se suprimieron los ítems cuyas cargas factoriales fueran inferiores a ,30, así como los que presentaban cargas superiores a ,30 en varios constructos.

Por otro lado, se realizó un AFC a partir de la solución factorial propuesta por el AFE. Para comprobar el ajuste global del modelo a través del AFC se utilizaron diversos índices de bondad de ajuste recomendados (HAIR et al. 2006; KLINE, 2005): el chi-cuadrado normalizado $\left(\chi^{2} / g l\right)$ que debe ser inferior a 3 (KLINE, 2005); el Error de Aproximación Cuadrático Medio (RMSEA) que debe presentar un valor inferior a ,08 (HU; BENTLER, 1999); el Índice de Ajuste No Normalizado (NNFI), el Índice de Ajuste Comparado (CFI) y el Índice de Ajuste Incremental (IFI), que deben presentar valores superiores a ,90 (HU; BENTLER, 1999).

Asimismo, se analizó la validez de constructo a través de la validez convergente y discriminante. En el caso del primer tipo de validez se observó la significación de las cargas factoriales $(p<, 05)$ de los indicadores en su respectiva dimensión y los valores de las pruebas $t$ asociados. Para contrastar la validez discriminante se observaron los coeficientes de correlación 
entre pares de factores, que deben ser inferiores a ,85 (KLINE, 1985) e inferiores a la raíz cuadrada de la varianza media extraída (AVE) (FORNELL; LARCKER, 1981).

Para comprobar la fiabilidad de la escala se utilizaron tres medidas: el alfa de Cronbach y la fiabilidad compuesta (FC), que deben mostrar valores superiores a .70 (HAIR et al., 2006), y la AVE que debe presentar valores superiores a .50 (FORNELL; LARCKER, 1981).

\section{RESULTADOS}

\subsection{ESTADÍSTICOS DESCRIPTIVOS}

En la tabla 1 se muestran la media, desviación típica, asimetría y curtosis de cada indicador. Si tenemos en cuenta que el valor neutral de la escala es 3 , la gran mayoría de ítems están por encima de esta media lo que indicaría una tendencia de los residentes al acuerdo de muchos de las variables de esta escala.

Dentro del factor de impacto sociocultural el ítem con una puntuación media más alta es el I-SC4 ( $M=4,28 ; \mathrm{DT}=, 84)$. En la dimensión de impacto en el desarrollo urbano y en las infraestructuras destaca el indicador I-DU1 ( $M=3,66$; $D T=1,11)$, aunque este factor también presenta bastantes indicadores con una puntuación media neutral o con ligera tendencia hacia el desacuerdo, como la observada en el ítem I-DU4 (M=2,86; DT=1,19). En el factor de impacto socioeconómico destacan los indicadores I-SE5 ( $M=3,91$; $D T=1,05=)$ e I-SE6 $(M=3,72 ; D T=1,13)$, mientras que en la dimensión de impacto en la imagen y la promoción del municipio destaca el indicador I-IM1 (M=4,07; DT=,94). En cuanto al factor de impacto en la cohesión social, igualdad y equidad sobresale el ítem I-Cl3 ( $M=3,90 ; \mathrm{DT}=, 96)$. Por último, en las dimensiones de impacto en la salud e impacto sobre los hábitos y niveles de actividad física destacan los indicadores I-SL3 ( $M=4,33$; $D T=, 85)$ e I-HA4 $(M=4,25 ; D T=, 88)$. Por otro lado, los valores de asimetría y curtosis son aceptables ya que son inferiores a 3.0 en todos los ítems (CHOU; BENTLER, 1995).

Tabla 1- Resultados descriptivos de la escala.

\begin{tabular}{|c|c|c|c|c|c|}
\hline Ítems & Variables & Media & DT & A & $C$ \\
\hline & Impacto sociocultural (I-SC) & & & & \\
\hline I-SC1 & $\begin{array}{l}\text { La implantación del centro deportivo de Moncada (CDM) ha } \\
\text { mejorado la solidaridad y la hospitalidad de los residentes } \\
\text { con los nuevos visitantes. }\end{array}$ & 3,73 & ,95 &,- 24 &,- 43 \\
\hline I-SC2 & $\begin{array}{l}\text { Favorece la preservación y conservación de la cultura del } \\
\text { pueblo. }\end{array}$ & 3,57 & 1,11 &,- 33 &,- 78 \\
\hline I-SC3 & $\begin{array}{l}\text { Favorece el intercambio cultural y la comprensión de otras } \\
\text { culturas. }\end{array}$ & 3,63 & 1,08 &,- 54 &,- 23 \\
\hline I-SC4 & $\begin{array}{l}\text { Proporciona a los ciudadanos una oportunidad para } \\
\text { conocer a nuevas personas. }\end{array}$ & 4,28 & ,84 & $-1,32$ & 2,09 \\
\hline \multirow[t]{2}{*}{ I-SC5 } & La apertura de CDM hace que el pueblo sea más seguro. & 3,07 & 1,22 & ,02 &,- 88 \\
\hline & $\begin{array}{l}\text { Impacto en el desarrollo urbano y en las infraestructuras } \\
\text { (I-DU) }\end{array}$ & & & & \\
\hline I-DU1 & $\begin{array}{l}\text { La apertura de CDM ayuda a la regeneración de las } \\
\text { infraestructuras de la zona. }\end{array}$ & 3,66 & 1,11 &,- 51 &,- 46 \\
\hline
\end{tabular}


Continuación...

I-DU2 Ha mejorado los servicios sociales en el pueblo. $\begin{array}{llll}3,54 & 1,09 & -, 35 & -, 49\end{array}$

I-DU3 Ha mejorado las infraestructuras en el pueblo (accesos, calles, mobiliario urbano...).

$\begin{array}{llll}3,27 & 1,19 & -, 21 & -, 79\end{array}$

I-DU4 Ha mejorado el sistema de transporte público. $\begin{array}{llll}2,86 & 1,19 & , 11 & -, 75\end{array}$

I-DU5 La apertura de CDM ha hecho que haya más interés por las viviendas del barrio

$\begin{array}{llll}3,13 & 1,22 & -, 04 & -, 89\end{array}$

I-DU6 La apertura de CDM ha revalorizado las viviendas del barrio

$\begin{array}{llll}3,16 & 1,16 & -, 03 & -, 75\end{array}$
Impacto socioeconómico (I-SE)

I-SE1 La apertura de CDM aumenta el negocio de los comercios y los negocios del pueblo.

$\begin{array}{llll}3,25 & 1,16 & -, 12 & -, 88\end{array}$

I-SE2

La apertura de CDM incrementa las oportunidades de trabajo.

$\begin{array}{llll}3,46 & 1,10 \quad-, 35 & -, 61\end{array}$

I-SE3 La apertura de CDM incrementa las inversiones privadas y las oportunidades de negocio.

$\begin{array}{llll}3,22 & 1,12 & -, 03 & -, 75\end{array}$

I-SE4 La apertura de CDM incrementa el consumo en el pueblo.

I-SE5 Debido a CDM ahora viene más gente al pueblo

$3,40 \quad 1,12 \quad-, 20 \quad-, 74$

I-SE6 La apertura de CDM aporta beneficios económicos al pueblo.

$\begin{array}{llll}3,91 & 1,05 & -, 69 & -, 31\end{array}$
Impacto en la imagen y la promoción del municipio (I-IM)

I-IM1 La apertura de CDM mejora la imagen del pueblo.

$\begin{array}{llll}3,72 & 1,13 & -, 60 & -, 47\end{array}$

I-IM2 La apertura de CDM facilita la apertura del barrio al resto del pueblo.

$\begin{array}{llll}4,07 & , 94 & -94 & , 94\end{array}$

I-IM3 La implantación de CDM aumenta el reconocimiento del pueblo.

$3,78 \quad, 96 \quad-, 46 \quad-, 19$

$\begin{array}{llll}3,86 & 1,01 & -, 62 & -, 15\end{array}$

Impacto en la cohesión social, igualdad y equidad (I-Cl)

I-Cl1 La apertura de CDM ha aumentado el sentido de orgullo por el pueblo

I-Cl2 Gracias a la implantación de CDM ha mejorado la cohesión social y la unión

$\begin{array}{lll}1,06 & -, 41 \quad-, 32\end{array}$

$\begin{array}{llll}3,56 & 1,04 & -, 31 & -, 48\end{array}$

I-Cl3 La apertura de CDM ha hecho que mucha gente mejore sus relaciones sociales

$3,90 \quad, 96 \quad-, 60 \quad-, 18$
Impacto sobre la salud (I-SL)

I-SL1 La apertura de CDM mejorará el bienestar general de los ciudadanos

I-SL2 Gracias a CDM mejora la salud general de los ciudadanos Creo que CDM influirá en unos mejores hábitos deportivos de los jóvenes

$\begin{array}{llll}4,06 & , 98 & -, 99 & , 57\end{array}$

La apertura de CDM mejora el bienestar psicológico en los

I-SL4 residentes

$\begin{array}{llll}4,15 & , 92 & -1,22 & 1,57\end{array}$

$\begin{array}{llll}4,33 & , 85 & -1,36 & 1,90\end{array}$

$\begin{array}{llll}4,10 & , 96 & -1,01 & , 73\end{array}$ Impacto sobre los hábitos y niveles de actividad física

\begin{tabular}{llllll}
\hline I-HA1 & $\begin{array}{l}\text { Mi interés por el deporte ha aumentado gracias a la } \\
\text { apertura de CDM }\end{array}$ & 4,02 & 1,07 & $-1,14$ & ,87 \\
I-HA2 & $\begin{array}{l}\text { La apertura de CDM ha hecho que la gente mejore su } \\
\text { cultura deportiva }\end{array}$ & 4,13 &, 90 & $-1,05$ & 1,11 \\
I-HA3 $\begin{array}{l}\text { Gracias a la apertura de CDM, los jóvenes ocupan mejor su } \\
\text { tiempo libre }\end{array}$ & 4,11 &, 94 &,- 91 &, 36 \\
$\begin{array}{l}\text { Creo que la apertura de CDM aumentará la práctica } \\
\text { I-HAportiva de los jóvenes }\end{array}$ & 4,25 &, 88 & $-1,05$ &, 62 \\
\hline
\end{tabular}




\subsection{ANÁLISIS FACTORIAL EXPLORATORIO}

En primer lugar, se realizó un análisis factorial exploratorio para los 31 ítems asociados a posibles impactos del centro deportivo siguiendo las recomendaciones mencionadas en el apartado de análisis de datos. Se utilizó el método MV para la extracción de factores, que es recomendable cuando la distribución de los ítems es aproximadamente normal (valores de asimetría y curtosis menores a -2 0 2) (LLORET-SEGURA et al., 2014). Se comprobó mediante el Análisis Paralelo que la solución factorial que mejor se ajustaba al objeto de estudio y que tenía una mejor interpretabilidad estaba compuesta por siete factores. No obstante, se eliminaron tres ítems por el incumplimiento de los criterios anteriormente expuestos debido a que presentaban unas cargas factoriales inferiores a .30 o superiores a .30 en dos o más factores. Los indicadores eliminados fueron los siguientes: I-SC5, I-DU1 y I-DU2.

Después de eliminar los tres ítems que incumplían los criterios expuestos, se realizó un nuevo análisis factorial que mostró un buen ajuste de la estructura factorial ya que el índice RMCR fue de .02, inferior al punto de corte recomendado (<.50), y el valor del índice GFI fue de .99 , superior al punto de corte recomendado (>.95). Los siete factores en los que se agruparon los 28 ítems restantes explicaron un $71.03 \%$ de la varianza. La solución factorial definitiva permitió observar que los indicadores retenidos quedaban englobados en el mismo factor que la propuesta inicial agrupados en las siguientes dimensiones: impacto sociocultural, impacto socioeconómico, impacto en la imagen y la promoción del municipio, impacto en el desarrollo urbano y las infraestructuras, impacto en la cohesión social, igualdad y equidad, impacto sobre la salud e impacto sobre los hábitos y niveles de actividad física.

\subsection{ANÁLISIS FACTORIAL CONFIRMATORIO}

La aplicación del AFC sobre la estructura factorial extraída del AFE previo mostró un buen ajuste, tal y como se puede observar en los índices de bondad de ajuste del modelo: chi-cuadrado significativo $\left(\chi^{2}=705,59 ; \mathrm{gl}=329 ; p<, 05\right)$ y un valor del chi-cuadrado normado $\left(\chi^{2}\right)$ $\mathrm{gl}=2,14$ ) inferior a 3 y el índice RMSEA mostró un valor de ,053 (IC=,049-,059), inferior a .08. En la misma línea, el resto de índices muestran un buen ajuste del modelo, ya que presentaban valores superiores a ,90: $\mathrm{NNFI}=, 94 ; \mathrm{CFI}=, 94 ;$ e $\mathrm{IFI}=, 94$.

Para comprobar la validez convergente de la escala se comprobó que las cargas factoriales de los ítems de cada constructo fueron significativas ( $t>1.96)$ ya que los valores de las pruebas t de todas las variables oscilaron desde 12.33 hasta 20.03. También presentaron valores superiores al punto de corte recomendado $(>, 60)$, tal y como se puede observar en la tabla 2. En cuanto a la validez discriminante, por un lado, comprobamos que todas las correlaciones entre los diversos factores fueron inferiores a .85 , cumpliéndose dicho criterio como se puede observar en la tabla 3; por otro lado, se comprobó que la raíz cuadrada del AVE era superior a la correlación entre pares de factores. 
Tabla 2 - Cargas factoriales, fiabilidad compuesta, medida de la varianza extraída y alfa de Cronbach de los indicadores de la escala.

\begin{tabular}{|c|c|c|c|c|c|}
\hline Ítems & & $\begin{array}{c}\text { Carga } \\
\text { factorial }\end{array}$ & $\alpha$ & FC & AVE \\
\hline & Impacto sociocultural (I-SC) & & 80 & ,80 &, 51 \\
\hline I-SC1 & & ,67 & & & \\
\hline I-SC2 & & ,72 & & & \\
\hline I-SC3 & & 85 & & & \\
\hline I-SC5 & & 83 & & & \\
\hline & Impacto en el desarrollo urbano y en las infraestructuras (I-DU) & & 85 & ,85 &, 60 \\
\hline I-DU3 & & ,67 & & & \\
\hline I-DU4 & & ,72 & & & \\
\hline I-DU5 & & 85 & & & \\
\hline I-DU6 & & 83 & & & \\
\hline & Impacto socioeconómico (I-SE) & &, 87 & 87 &, 53 \\
\hline I-SE1 & & ,74 & & & \\
\hline I-SE2 & & ,72 & & & \\
\hline I-SE3 & & ,73 & & & \\
\hline I-SE4 & & 81 & & & \\
\hline I-SE5 & & ,63 & & & \\
\hline I-SE6 & & ,74 & & & \\
\hline & Impacto en la imagen y la promoción del municipio (I-IM) & & 84 & 84 &, 64 \\
\hline I-IM1 & & ,82 & & & \\
\hline I-IM2 & & ,75 & & & \\
\hline I-IM3 & & 83 & & & \\
\hline & Impacto en la cohesión social, igualdad y equidad (I-Cl) & & 82 & ,82 &, 61 \\
\hline $\mathrm{I}-\mathrm{Cl} 1$ & & ,78 & & & \\
\hline $\mathrm{I}-\mathrm{Cl} 2$ & & ,82 & & & \\
\hline $\mathrm{I}-\mathrm{Cl} 3$ & & ,74 & & & \\
\hline & Impacto sobre la salud (I-SL) & & 87 & 87 &, 63 \\
\hline I-SL1 & & 80 & & & \\
\hline I-SL2 & & 81 & & & \\
\hline I-SL3 & &, 80 & & & \\
\hline I-SL4 & & ,76 & & & \\
\hline & Impacto sobre los hábitos y niveles de actividad física & & 86 & ,86 &, 62 \\
\hline I-HA1 & & ,64 & & & \\
\hline I-HA2 & & 82 & & & \\
\hline I-HA3 & & 85 & & & \\
\hline I-HA4 & & 82 & & & \\
\hline
\end{tabular}

Nota: $\alpha=$ alfa de Cronbach; FC=Fiabilidad compuesta; AVE=Varianza media extraída

Fuente: Elaboración propia.

La fiabilidad del modelo se contrasto a través del alfa de Cronbach, FC y AVE. Tal y como se puede comprobar en la tabla 2, los coeficientes del Alfa de Cronbach como los de la FC para cada factor oscilaron entre, 80 y, 87 , siendo superiores al criterio recomendado $(>, 70)$. Asimismo, los valores de la AVE oscilaron entre ,51 y ,64, también superiores al punto de corte recomendado $(>, 50)$. 
Tabla 3. Matriz de correlaciones interfactorial de la escala derivada del AFC.

\begin{tabular}{|c|c|c|c|c|c|c|c|}
\hline & I-SC & I-DU & I-SE & I-IM & $\mathrm{I}-\mathrm{Cl}$ & I-SL & I-HA \\
\hline I-SC & .71 & & & & & & \\
\hline I-DU &, $58^{* *}$ & .77 & & & & & \\
\hline I-SE &, $56 *$ & 68* & .73 & & & & \\
\hline I-IM &, $55^{* *}$ & , $48^{* * *}$ &, $63^{* *}$ & .80 & & & \\
\hline $\mathrm{I}-\mathrm{Cl}$ & ,67" &, $52^{* *}$ &, $59^{* *}$ & ,68** & .78 & & \\
\hline I-SL & ,48*" & , $30^{* *}$ &, $48^{* *}$ & ,66" & ,64** & .80 & \\
\hline I-HA & ,47* & ,32** &, $43^{*}$ & ,62** &, $61^{* *}$ & ,70** & .79 \\
\hline
\end{tabular}

Nota. I-SE=Impacto sociocultural; I-DU=Impacto en las infraestructuras y el desarrollo urbano; I-SE=Impacto socioeconómico; I-IM=Impacto en la imagen y la promoción del municipio; I-Cl=Impacto en la cohesión social, igualdad y equidad; I-SL=Impacto sobre la salud; I-HA=Impacto sobre los hábitos y niveles de actividad física; " $p \leq .01$; en la diagonal se muestran los valores de la raíz del AVE para cada factor. Fuente: Elaboración propia.

\section{DISCUSIÓN Y CONCLUSIONES}

En este estudio se ha desarrollado y validado una escala que trata de medir las percepciones de los residentes acerca de los impactos de una instalación deportiva, situada en dicha localidad. Como se ha comentado, no existe en la literatura, trabajos que analicen los impactos sociales que causan las instalaciones deportivas dentro de una población. Bien es cierto que en la literatura encontramos diversidad de trabajos que analizan los impactos sociales de eventos deportivos o de turismo. Por ello, este estudio, trata de validar una escala que sirva para poder conocer las percepciones de los residentes de un municipio acerca del impacto de una instalación deportiva, y con ello poder aportar herramientas a la gestión del deporte.

El AFE y AFC que se realizó sobre las percepciones de los residentes de la localidad, mostraban una adecuada validez y fiabilidad de la muestra utilizada, como se puede observar en el apartado anterior. No obstante para poder ajustar más el instrumento utilizado, se eliminaron 3 ítems por incumplimiento de criterios, quedando finalmente un instrumento estructurado de 28 ítems, compuesto por 7 dimensiones.

La primera dimensión se refiere al impacto sociocultural, compuesta por 5 ítems, relacionados con la solidaridad, el intercambio cultural y la seguridad. Estos ítems han sido analizados en otros trabajos de esta área de investigación relacionados con el interés cultural (BALDUCK; MAES; BUELENS, 2011), impactos socioculturales (CALABUIG et al., 2014; PARRA et al., 2016; PRAYAG et al., 2013) y los beneficios sociales (LLOPIS; GIL, 2011). Los ciudadanos conocen el efecto socializador que tiene la práctica deportiva, y entienden que, acudir al centro deportivo facilitará las relaciones sociales, así como la apertura de la instalación implicará mayor tráfico y tránsito de personas, por lo que habrá más vigilancia, entendiendo así un aumento de la seguridad de la población. El hecho también, que dentro de la misma localidad se encuentre una universidad privada con un elevado porcentaje de estudiantes extranjeros que también acuden al centro deportivo, permite a los residentes entender que la instalación deportiva favorece el intercambio cultural.

La segunda dimensión se identifica con el impacto en el desarrollo urbano y las infraestructuras, formado por ítems que se engloban en las infraestructuras como el mobiliario urbano, el transporte o la revalorización de las viviendas del municipio. Esta dimensión, es 
analizada en otros estudios en la que ha sido consultada en el contexto de los eventos deportivos (LLOPIS; GIL, 2011; GÓNZALEZ- GARCÍA et al., 2016; PARRA et al., 2016). Esta dimensión ha sido evaluada por los ciudadanos como consecuencia de los cambios estructurales que ha sufrido la población en la zona donde está ubicada la instalación con mejoras del pavimentado como de los accesos, también se ha ofrecido a los ciudadanos una ruta con el autobús urbano, que es gratuito, para poder llegar a la instalación deportiva, desde todos los puntos de la población.

Como tercera dimensión, se identifica el impacto socioeconómico con 6 ítems relacionados con los negocios como los comercios, las oportunidades de trabajo, el consumo y los beneficios económicos. Se han encontrado trabajos que identifican esta dimensión dentro de los eventos deportivos (BALDUCK, MAES; BUELENS, 2011; KIM et al., 2006; LIU, 2016). Se observa como las percepciones de los residentes hacia una mayor afluencia de ciudadanos de otros pueblos, es debido a la instalación deportiva, y es que en una ratio de $15 \mathrm{~km}$, no se encuentra otra instalación de similares características. También, debido a la diversidad de servicios que ofrece el centro deportivo (natación para niños, embarazadas, tercera edad, etc.) hay una gran afluencia de personas provenientes de otras poblaciones y, en consecuencia, un mayor consumo en la población donde esta ubicado el centro deportivo, así como la generación de oportunidades de trabajo para los residentes del municipio.

La cuarta dimensión hace referencia a la imagen y promoción del municipio, identificada con 3 ítems, que se relacionan con la mejora de la imagen y del reconocimiento del municipio con respecto a la instalación. Al igual que en las demás dimensiones, se encuentran trabajos que también identifican esta dimensión en el contexto de los eventos deportivos (CHEN; TIAN, 2015; PARRA et al., 2016). Los residentes valoran positivamente que la apertura de la instalación deportiva influye en la imagen del pueblo, partiendo que esta instalación posee en su nomenclatura el nombre de la población, así también, el hecho de no encontrar en municipios cercanos, instalaciones de tales características, otorga a la población un valor añadido en cuanto a su promoción e imagen.

La quinta dimensión definida identifica el impacto en la cohesión social, igualdad y equidad compuesta por 3 ítems que refieren al orgullo del municipio, la cohesión y las relaciones sociales. Ítems que guardan relación con trabajos en el ámbito de los eventos deportivos (GURSOY et al., 2011; KIM et al., 2015). Los residentes se sienten orgullosos de su municipio gracias a la instalación deportiva, y es que este centro deportivo ha dotado a la población de un servicio deportivo de gran calado, situando a la localidad en un lugar de privilegio con respecto a otros municipios. Así mismo, los residentes valoran que se den contextos sociales favorables con la apertura del centro deportivo, debido a las interacciones sociales que se producen en este, por la diversidad de actividad física, los campeonatos que se realizan, etc.

En referencia a la sexta dimensión, relacionado con el impacto en la salud, existen diferentes estudios (KAPLANIDOU; KARADAKIS, 2010; KARADAKIS; KAPLANIDOU, 2012; LEOPKEY; PARENT, 2012) en los que la dimensión identificada con la salud junto con otros factores es percibida de manera considerable dentro de los legados de los eventos deportivos. Siendo esta la dimensión con la valoración media más elevada, los residentes conocen los múltiples beneficios que tiene la práctica deportiva para la salud tanto a nivel físico como psicológico de los practicantes, y entienden que el centro deportivo favorecerá los hábitos deportivos de los jóvenes, ya que son un perfil sociodemográfico, con mucho tiempo libre y fácil de influenciar, evitando así malas conductas. 
En cuanto a la última dimensión relacionada con los hábitos y niveles de actividad física donde los ítems miden el interés por el deporte de los residentes con la apertura de la instalación deportiva, la cultura deportiva de los mismos, y la práctica deportiva y tiempo libre de los jóvenes de la localidad. Se encuentran similitudes con otros trabajos que evidencian dichas percepciones en el contexto de los eventos deportivos (MA et al., 2011; GONZÁLEZ-GARCÍA et al., 2016). Esta dimensión también ha sido una de las mejores valoradas por los ciudadanos, entendiendo los residentes que, con la instalación deportiva y la actividad física realizada en ella, contribuye a mejorar hábitos y dotar a los usuarios, y a la población en general, de mejores niveles de actividad física.

Como conclusión, el instrumento validado para medir las percepciones de los residentes del municipio sobre el impacto social de las instalaciones deportivas presenta un buen ajuste y puede aportar a la gestión del deporte una herramienta útil, capaz de dar información social a los estamentos o a los distintos gestores deportivos. Así como en futuras investigaciones utilizar dicha escala en otros contextos en los que se encuentran diferentes instalaciones deportivas. Como posibles limitaciones y probables líneas de investigación futuras, recalcar que no existe en la literatura trabajos con los que poder contrastar los resultados de este estudio y que analicen las percepciones de residentes acerca del impacto social de instalaciones deportivas. Debe considerarse, que el muestreo es de conveniencia, es decir, se deben tomar con prudencia ya que no se puede universalizar a toda la población. Un dato que también aportaría a la mejor comprensión de las percepciones de los residentes es conocer el nivel de ingresos de cada residente.

\section{REFERENCIAS}

AÑÓ, Vicente; CALABUIG, Ferran; PARRA, David. Impacto social de un gran evento deportivo: el Gran Premio de Europa de Fórmula 1. Cultura, Ciencia y Deporte, v. 7, n. 19, p. 53-65, mar. 2012.

ATKINSON, Jennifer L; SALLIS, James F; SAELENS, Brian. E; CAIN, Kelly. L; BLACK, Jennifer. $B$. The association of neighborhood design and recreational environments with physical activity. American Journal of Health Promotion, v. 19, n. 4, p. 304-309, mar./abr. 2005.

BALDUCK, Anne-Line; MAES, Marc; BUELENS, Marc. The social impact of the tour de France: Comparisons of residents' pre-and post-event perceptions. European Sport Management Quarterly, v. 11, n. 2, p. 91-113, may 2011.

BOONE, Louis E; KURTZ, David. L. Contemporary Marketing. New York: Harcourt College, 2012.

BROWN, Adam; MASSEY, Joanne. The sports development impact of the Manchester 2002 Commonwealth Games: Post Games Report. London: UK Sport, 2004.

CALABUIG, Ferran; PARRA, David; AÑO, Vicente; AYORA, Daniel. Análisis de la percepción de los residentes sobre el impacto cultural y deportivo de un Gran Premio de Fórmula 1.

Movimento, v. 20, n. 1, p. 261-280, ene./mar. 2014. 
CHEN, Fangying; TIAN, Lichao. Comparative study on residents' perceptions of follow-up impacts of the 2008 Olympics. Tourism Management, v. 51, p. 263-281, dic. 2015.

CHENG, Elaine; JARVIS, Nigel. Residents' perception of the social-cultural impacts of the 2008 Formula 1 Singtel Singapore Grand Prix. Event Management, v. 14, n. 2, p. 91-106, 2010.

CHOU, Chih-Ping; BENTLER, Peter. Estimates and tests in structural equation modeling. In: HOYLE, R. H. (Ed.), Structural equation modeling: Concepts, issues, and applications. Thousand Oaks: Sage, 1995. p. 37-55.

COLLINS, Andrea; FLYNN, Andrew; MUNDAY, Max; ROBERTS, Annette. Assessing the environmental consequences of major sporting events: The 2003/04 FA Cup Final. Urban Studies, v. 44, n. 3, p. 457-476, mar. 2007.

DECCIO, Cary; BALOGLU, Seyhmus. Non-host community resident reactions to the 2002 Winter Olympics: The spillover impacts. Journal of Travel Research, v. 41, n. 1, p. 46-56, ago. 2002.

DISHMAN, Rod K; SALLIS, James F; ORENSTEIN, Diane. R. The determinants of physical activity and exercise. Public Health Reports, v. 100, n. 2, p. 158-171, mar./abr. 1985.

FORNELL, Claes; LARCKER, David. Evaluating structural equation models with unobservable variables and measurement error. Journal of Marketing Research, v. 18, n.1, p. 39-50, feb. 1981.

FREDLINE, Liz; JAGO, Leo; DEERY, Margaret. The development of a generic scale to measure the social impacts of events. Event Management, v. 8, n. 1, p. 23-37, ene. 2003.

GIBSON, Heather. Sport tourism: concepts and theories. New York: Routledge, 2006. GONZÁLEZ-GARCÍA, Rómulo; PARRA, David; AÑÓ, Vicente; CALABUIG, Ferran. Percepción de los residentes sobre el impacto del Mundobasket 2014 en Gran Canaria y apoyo a la celebración de eventos deportivos. Revista Iberoamericana de Psicología del Ejercicio y el Deporte, v. 11, n. 2, p. 279-288, mar. 2016.

GURSOY, Dogan; KENDALL, Ken. Hosting mega events: Modeling locals' support. Annals of Tourism Research, v. 33, n. 3, p. 603-623, jul.2006.

GURSOY, Dogan; CHI, Cristina; Al, Jin; CHEN, Brendan. Temporal Change in Resident Perceptions of a Mega-Event: The Beijing Olympic Games. Tourism Geographies, v. 13, n. 2, p. 299-324, jun. 2011.

HAIR, Joseph; BLACK, William; BABIN, Barry; ANDERSON, Rolph; TATHAM, Ronald. Multivariate data analysis. New Jersey: Prentice Hall, 2006.

HALL, Colin. Hallmark tourist events: impact, management, and planning. London: Belhaven, 1992.

HARMAN, Harry. Análisis factorial moderno. Madrid: Saltés, 1980.

HILLER, Harry. Post-event Outcomes and the Post-modern Turn: The Olympics and Urban Transformations. European Sport Management Quarterly, v. 6, n. 4, p. 317-332, ene. 2006.

HU, Li-tze; BENTLER, Peter. Cutoff criteria for fit indexes in covariance structure analysis: Conventional criteria versus new alternatives. Structural Equation Modeling: A 
KAPLANIDOU, Kiki; KARADAKIS, Kostas. Understanding the Legacies of a Host Olympic City: The Case of the 2010 Vancouver Olympic Games. Sport Marketing Quarterly, v. 19, n. 2, p. 110-117, ene. 2010.

KARADAKIS, Kostas; KAPLANIDOU, Kiki. (2012). Legacy Perceptions among Host and Nonhost Olympic Games Residents: A Longitudinal Study of the 2010 Vancouver Olympic Games. European Sport Management Quarterly, v. 12, n. 3, p. 243-264, may. 2012.

KIM, Hyun; GURSOY, Dogan; LEE, Soo. The impact of the 2002 World Cup on South Korea: comparisons of pre- and post-games. Tourism Management, v. 27, n. 1, p. 86-96, feb. 2006.

KIM, Wonyoung; JUN, Ho; WALKER, Matthew; DRANE, Dan. Evaluating the perceived social impacts of hosting large-scale sport tourism events: scale development and validation. Tourism Management, v. 48, p. 21-32, jun. 2015.

KLINE, Rex. Principles and practice of structural equation modeling. $2 .^{\text {nd }}$ ed.. New York: Guilford, 2005.

LEOPKEY, Becca; PARENT, Milena. Olympic Games legacy: From general benefits to sustainable long-term legacy. International Journal of the History of Sport, v. 29, n. 6, p. 924-943, abr. 2012.

LIM, So Youn; WARNER, Stacey; DIXON, Marlene; BERG, Brennan.; KIM, Chiyoung; NEWHOUSE-BAILEY, Michael. Sport participation across national contexts: A multilevel investigation of individual and systemic influences on adult sport participation. European Sport Management Quarterly, v. 11, n. 3, p. 197-224, jun. 2011.

LIU, Dongfeng. Social impact of major sports events perceived by host community. International Journal of Sports Marketing and Sponsorship, v. 17, n. 1, p. 78 -91, ene. 2016.

LLOPIS, María; GIL, Irene. Un gran evento deportivo: perspectiva de los residentes de la ciudad de acogida. Gran Tour, revista de investigaciones turísticas, v. 4, p. 32-61, ene. 2011.

LLORET-SEGURA, Susana; FERRERES-TRAVER, Adoración; HERNÁNDEZ-BAEZA, Ana; TOMÁS-MARCO, Inés. El análisis factorial exploratorio de los ítems: una guía práctica, revisada y actualizada. Anales de Psicología, v. 30, n. 3, p. 1151-1169, sep-dic 2014.

LORDE, Troy; GREENIDGE, Dion; DEVONISH, Dwayne. Local resident's perceptions of the impacts of the ICC Cricket World Cup 2007 on Barbados: Comparisons of pre- and post-games. Tourism Management, v. 32, n. 2, p. 349-356, abr. 2011.

LORENZO-SEVA, Urbano; FERRANDO, Pere. FACTOR: a computer program to fit the exploratory Factor Analysis model. Behavioral Research Methods, Instruments \& Computers, v. 3, n. 1, p. 88-91, mar. 2006.

MA, Shang; ROTHERHAM, lan; EGAN, David; MA, Shang. A framework for monitoring during the planning stage for a sports mega-event. Journal of Sustainable Tourism, v. 19, n. 1, p. 1-18, ene. 2011.

MALFAS, Maximos; THEODORAKI, Eleni; HOULIHAN, Barrie. Impacts of the Olympic Games as megaevents. Municipal Engineer, v. 157, n. 3, p. 209-220, ene. 2004.

NTLOKO, Ncedo; SWART, Kamilla. Sport tourism event impacts on the host community: a case of Red Bull Big Wave Africa. South African Journal for Research in Sport, Physical Education and Recreation, v. 30, n. 2, p. 79-93, sep. 2008. 
OLSEN, Marvin; MERWIN, Donna. Towards a methodology for conducting social impacts assessments using quality of life indicators. In: FINSTERBUSCH, K.; WOLF, C. (Eds.). Methodology of social impact assessment. Pennsylvania: Dowden, Hutchinson \& Ross, 1977.

PARRA, David; AÑÓ, Vicente; CALABUIG, Ferran; AYORA, Daniel. Percepción de los residentes sobre el legado de la America's Cup. Cuadernos de Psicología del Deporte, v. 16, n. 1, p. 309-322, ene. 2016.

PARRA, David; ELASRI, Amal; TRIADÓ, Xavier; APARICIO, Pilar. Análisis de la relación entre los beneficios y los costes percibidos y la satisfacción del residente con la celebración de un evento deportivo: efecto mediador del valor percibido. Revista de Psicología del Deporte, v. 25, n. 3, p. 59-63, jul. 2016.

PARRA, David; GONZÁLEZ, Rómulo; AÑÓ, Vicente; AYORA, Daniel. Percepción de los visitantes sobre el impacto social y sus intenciones con respecto a la celebración de un evento deportivo de pequeña escala. Revista de Psicología del Deporte, v. 25, n. 3, p. 93-96, jul. 2016.

PRAYAG, Girish; HOSANY, Sameer; NUNKOO, Robin; ALDERS, Taila. London residents support for the Olympic Games: The mediating effect of overall attitude. Tourism Management, v. 36, p. 629-640, jun. 2013.

PREUSS, Holger; SOLBERG, Henry Anne. Attracting major sporting events: The role of local residents. European Sport Management Quarterly, v. 6, n. 4, p. 391-411, 2006.

REISINGER, Yvette. International Tourism: Cultures and Behaviour. Oxford: ButterworthHeinemann, 2009.

RUIZ, Miguel Ángel; PARDO, Antonio; SAN MARTíN, Rafael. Modelos de ecuaciones estructurales. Papeles del psicólogo, v. 31, n. 1, p. 34-45, ene-abr. 2010.

SMITH, Andrew; FOX, Tim. From 'Event-led' to 'Event-themed' Regeneration: The 2002 Commonwealth Games. Urban Studies, v. 44, n. 5-6, p. 1125-1143, may. 2007.

SWART, Kamilla; BOB, Urmilla. Listening To Community Voices: Athlone and Green Point Residents Views on the Location of the 2010 FIFA World Cup Stadium in Cape Town. In: BÜCH, M.P.; MAENNIG, W.; SCHULKE, H.J. (Eds). Zur Ökonomik von Spitzenleistungen im internationalen Sport. Hamburg: Hamburg University, , 2012. p. 101-122.

TANAKA, Jeffrey; HUBA, George. A general coefficient of determination for covariance structure models under arbitrary GLS estimation. British Journal of Mathematical and Statistical Psychology, v. 42, n. 2, p. 233-239, nov. 1989.

WAITT, Gordon. Social impacts of the Sydney Olympics. Annals of Tourism Research, v. 30, n. 1, p. 194-215, ene. 2003.

WAH, Kim Whatt Clifford; PYUN, Do-Young. Impact of sport facility upgrade on patron utilisation rate. Asian Journal of Physical Education and Sport Science, v. 3, n. 2, p. 41-56, 2015.

WEED, Mike; COREN, Esther; FIORE, Jo. A systematic review of the evidence base for developing a physical activity and health legacy from the London 2012 Olympic and Paralympic games. Canterbury, SPEAR, 2009. 
WICKER, Pamela; BREUER, Christoph; PAWLOWSKI, Tim. Promoting sport for all to agespecific target groups: The impact of sport infrastructure. European Sport Management Quarterly, v. 9, n. 2, p. 103-118, feb. 2009.

WICKER, Pamela; HALLMANN, Kristin; BREUER, Cristoph. Analyzing the impact of sport infrastructure on sport participation using geo-coded data: Evidence from multi-level models. Sport Management Review, v. 16, n. 1, 54-67, feb. 2013.

WILMSHURST, Jhon; MACKAY, Adrian. The Fundamentals and Practice of Marketing. London, Butterworth -Heinemann, 2002.

ZHOU, Yong; AP, Jhon. Residents' perceptions towards the impacts of the Beijing 2008 Olympic Games. Journal of Travel Research, v. 48, n. 1, p. 78-91, ago. 2009.

Apoio:

Universidad de Valencia 